\title{
287 Total pharyngolaryngectomy and oesophagectomy
}

A Gastric transposition necessitates cervical, thoracic and abdominal incisions.

B Colon transposition has the disadvantage of three anastamoses.

C Stenosis is the main complication of using stomach for the repair.

D Postoperative thyroid and calcium supplements are required.

E A pneumothorax is treated by insertion of a chest drain.

\section{Recurrence of hypopharyngeal carcinoma}

A Distant metastases are the usual cause of death.

B Regular follow-up examination provides reliable early detection.

C Possible causes include tumour implantation at operation.

D Stomal recurrence can be treated by thoracotracheostomy with manubrial resection, but is rarely successful.

E May present as a persistent pharyngocutaneous fistula.

\section{Malignant cervical lymph nodes}

A Assessment for TNM staging by palpation is subject to interobserver variation of around $30 \%$ among trained specialists.

B Palpable nodes not infrequently contain no tumour.

C Non-palpable nodes not infrequently contain tumour.

D Nodes in the supraclavicular fossa carry a worse prognosis than nodes in the submandibular triangle.

E Because of the unreliability of assessment, $\mathrm{N}$-status is unrelated to prognosis. 